Research Paper

\title{
High performance quantum computing
}

\author{
Simon J. DEVITT ${ }^{1}$, William J. MUNRO ${ }^{2}$, and Kae NEMOTO ${ }^{3}$ \\ ${ }^{1,3}$ National Institute for Informatics \\ ${ }^{2}$ NTT Basic Research Laboratories
}

\begin{abstract}
The architecture scalability afforded by recent proposals of a large-scale photonic-based quantum computer allows us to move on to a discussion of massively scaled Quantum Information Processing (QIP). In this paper, we consider the quantum analogue of High Performance Computing (HPC), where a dedicated server farm is utilized by many users to run algorithms and share quantum data. We introduce both a trusted mainframe model as a quantum extension of classical HPC and a model where quantum security is achieved during computation. The scaling structure of the optical architecture leads to an attractive future for server based QIP, where dedicated mainframes can be constructed and/or expanded to serve an increasingly hungry user base with the ideal resource for individual quantum information processing.
\end{abstract}

KEYWORDS

Quantum computing, topological clusters, High Performance Computing, secure computing

\section{Introduction}

Since the introduction of quantum information science in the late 1970's and early 1980's, a large scale physical device capable of high fidelity quantum information processing (QIP) has been a major and highly sought after goal. While quantum information has lead to many extraordinary developments in foundational quantum theory, quantum atom/optics, solid state physics and optics many researchers, world wide, are still striving towards developing a large scale, quantum computer.

The issue of computational scalability for QIP has been an intensive area of research for not only physicists but also computer scientists, mathematicians and network analysts and in the past decade have been many proposals for scalable quantum devices for a variety of quantum architectures [1]-[9]. The complexity in designing a large scale quantum computer is immense and research in this area must incorporate complex ideas in theoretical and experimental physics, information the-

Received November 1, 2010; Accepted January 4, 2011.

1) devitt@nii.ac.jp, ${ }^{2)}$ bill.munro@me.com, ${ }^{3)}$ nemoto@nii.ac.jp DOI: $10.2201 / \mathrm{NiiPi} .2011 .8 .6$ ory, quantum error correction, quantum algorithms and network design. Due to the relative infancy of theoretical and experimental QIP it has been difficult to implement theoretically scalable ideas in quantum information theory, error correction and algorithm design into an architectural model where the transition from 1-100 qubits to $1-100$ million qubits is conceptually straightforward.

Recent theoretical advancements in computational models for QIP has introduced an extremely elegant pathway to realize an enormously large QIP system in optics. Topological cluster state computing, first introduced by Raussendorf, Harrington and Goyal [10]-[12] has emerged as an extremely promising computational model for QIP. Integration of this model with chipbased photon/photon gates such as the photonic module [13] has lead to a promising optical realization of a quantum computer [5], Fig. 1. The conceptual scalability of the chip based topological computer allows, for the first time, a grounded discussion on large scale quantum information processing, beyond the individual computer. In this paper we take the scalability issue one step further, examining the possible long term imple- 


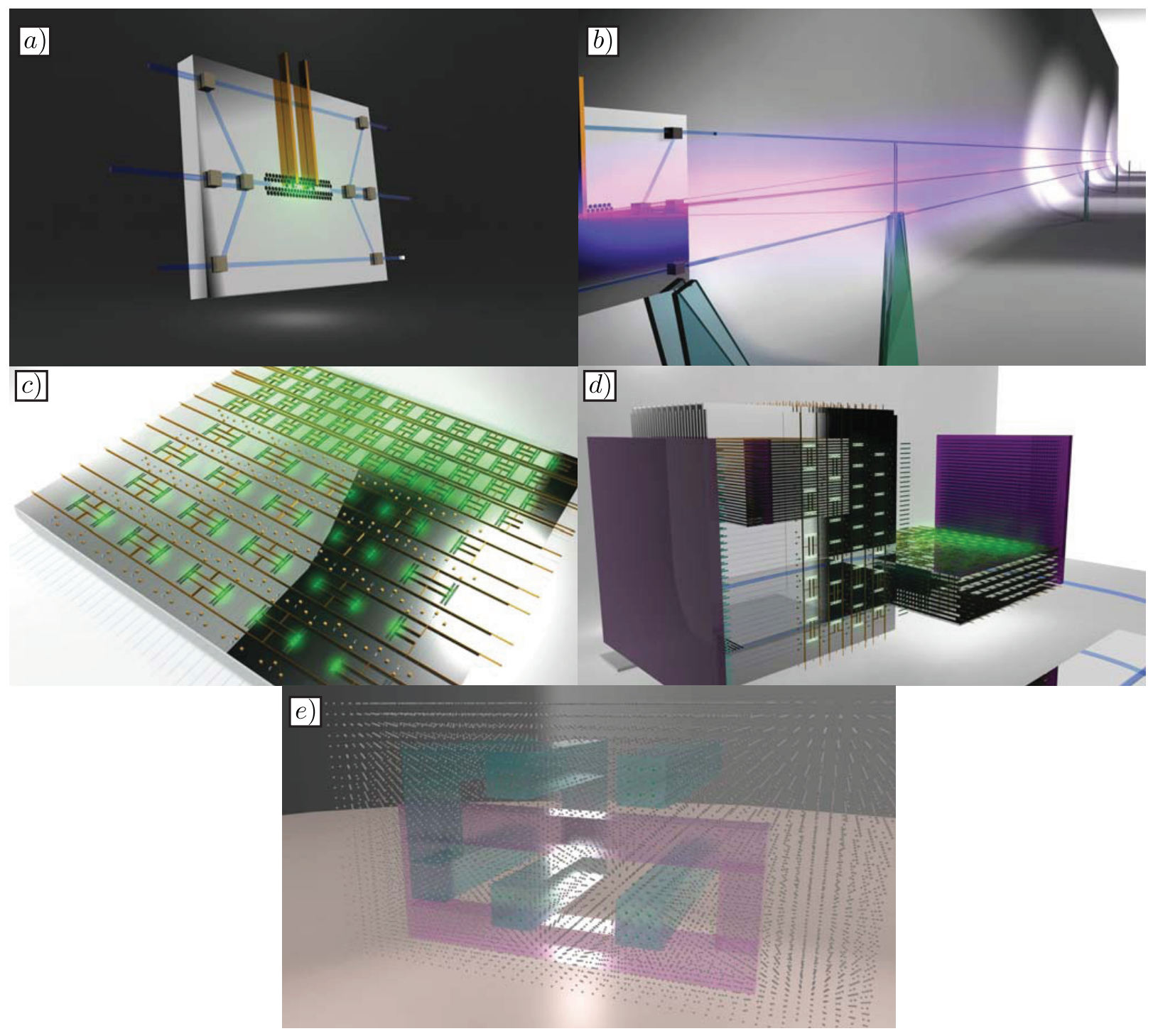

Fig. 1 Structure and components for an optics based topological quantum computer. a) The photonic module [13], an atom/cavity based device which is utilised to couple single photons. b) Photons sequentially move through the module and become entangled with the same single atom. Once the atom is measured, they are disentangled from the atom, but remain entangled to each other. c). Single wafter of coupled photonic modules which are used to construct a highly entangled cluster state to perform topological computation. d) actual quantum computer. Sets of wafers are stacked to produce the entangled photonic resource state, the computer gets bigger by simply adding more and more wafters. e). Once photons are entangled into a large 3D cluster state, they are measured to perform computation. Shown is a braided CNOT operation [10]-[12] incorporating full, fault-tolerant error correction.

mentation of topological cluster state computing with the photonic chip and discuss what the future may hold for this architectural model of QIP.

\section{The HPQC}

Traditional discussions of scalability in QIP is generally limited to the issue of constructing a single, mod- erately large scale quantum computer, capable of performing non-trivial algorithms for a single user. In the case of the optical topological computer [5] we can consider the possibility of mainframe computers and start to consider the quantum analogue of classical high performance computing, namely High Performance Quantum Computing (HPQC); where a large, generic quan- 


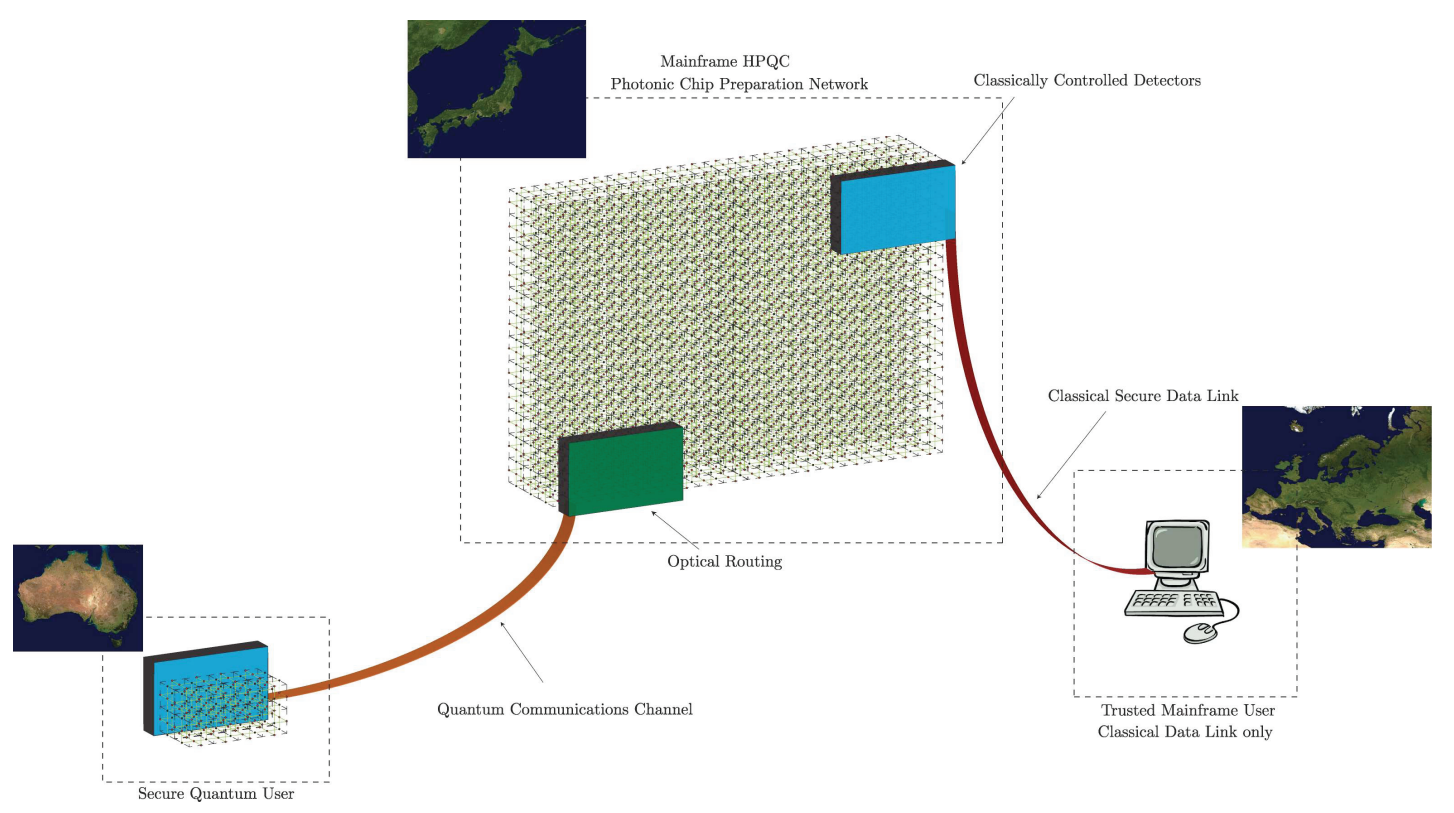

Fig. 2 A central mainframe HPQC would consist of a massive cluster preparation network built from single photons sources and photonic chips. Once the cluster is prepared, users can log on and perform individual computations in one of two ways. A trusted mainframe model is where the user submits a classical data stream corresponding to the measurement pattern for a quantum algorithm. The secured quantum user has access to a high fidelity quantum communications link between themselves and the mainframe. The allotted portion of the global lattice is then physically routed to the user and photon measurements are performed locally.

tum resource is made available to multiple clients to perform independent (or joint) QIP.

The topological computer is uniquely suited to this task, for several reasons. Aside from the error correcting and resource benefits of the topological cluster model, the basic geometric structure of the lattice allows for multi-user computation that would be problematic when utilizing the more traditional 2D cluster state techniques [14]. In traditional 2D cluster state computing, one dimension of the cluster represents "algorithmic qubits" while the other dimension represents simulated time. As one of the two dimensions of the cluster is simulated time, the arrangement of algorithmic qubits forms an effective Linear Nearest Neighbour (LNN) network. Therefore, if multiple users are sharing a common $2 \mathrm{D}$ cluster state, they could not interact data with each other or with a central resource core without transporting quantum information through parts of the cluster dedicated to other users.

Moving to topological clusters convert this LNN network topology into a 2D grid, enabling the partitioning of the cluster lattice into user regions and resource regions. Additionally, as the lattice is carried by single photons, we can potentially integrate a mainframe model with developments in quantum communications and entanglement distribution [15]-[17]. This gives a layer of security to the HPQC which would be difficult, if not impossible to achieve for multi-user, matter based qubit architectures.

Here we introduce the basic framework for a potential HPQC based on topological cluster states, computing in the photonic regime (Fig. 2). We discuss two possible mainframe models, one where multi-user computation is performed locally by the mainframe and another where partitions of the mainframe lattice are sent via quantum communications channels to individual users. We complete the discussion by providing a example of a partition structure for the mainframe lattice which satisfies many of the necessary components for a HPQC and give a basic estimate of the number of photonic chips required for a massive quantum server.

The first model we consider is denoted the trusted mainframe model. This is where individual users connect via classically secure data pathways and the mainframe host is trustworthy. Each client logs onto the host and transmits a classical data stream, corresponding to the desired quantum algorithm, to the host (via a sequence of photon measurement bases). The mainframe will then run the quantum algorithm locally and once the computation is complete, transmits the result- 
ing classical information back to the user.

This model has very substantial benefits. First, each user does not require quantum communications channels or any quantum infrastructure locally. All that is required is that each user compile a quantum algorithm into an appropriate classical data stream which is sent to the mainframe. The host does not need to transmit any data to the user during computation. All internal corrections to the lattice which arise due to its preparation and error correction procedures are performed within the mainframe. The only data transmitted to the user is the classical result from the quantum algorithm. Finally, as each user independently logs on to the system to run a quantum algorithm, the mainframe can be configured to assign resources dynamically. If one user requires a large number of logical qubits and if the mainframe load is low, then the host can adjust to allocate a larger partition of the overall lattice to one individual user.

While the user/mainframe interaction of this model is identical to classical models for high performance computing, the fact that we are working with qubits suggests the possibility of secure HPQC. In the trusted mainframe model the classical data stream from the user to host is susceptible to interception (although quantum key distribution and secure data links can be utilized to mitigate this issue) and the quantum mainframe has complete access to both the quantum algorithm being run on the server and the results of the computation. If sensitive computation is required, we can combine the mainframe with high fidelity communication channels to perform a secure version of HPQC in a manner unavailable to classical distributed computing.

As the topological lattice prepared by the mainframe is photon based, we are able to utilize high fidelity optical communications channels to physically transmit a portion of the $3 \mathrm{D}$ lattice to the client. Compared with the trusted mainframe model, this scheme has some technological disadvantages. High fidelity quantum communication channels are required to faithfully transmit entangled photons from the mainframe to each client. While purification protocols could, in principal, be utilized to increase transmission fidelity, this would be cumbersome and given that topological models for QIP exhibit very high thresholds (of the order of 0.1$1 \%$ ) it is fair to assume that communication channels will be of sufficient reliability when a mainframe device is finally constructed. Secondly, each client must have access to a certain amount of quantum technology. Specifically, a set of classically controlled, high fidelity single photon, wave-plates and detectors. This allows each client to perform their own measurement of the photon stream to perform computation locally.

Security arises as the quantum data stream never car- ries information related to the quantum algorithm being run on the client side. As the photon stream transmitted to the client is the $3 \mathrm{D}$ topological lattice generated by the mainframe, interrogation of the quantum channel is unnecessary as the state transmitted is globally known. Additionally, the only classical information sent between mainframe and user is related to the initial eigenvalues of the prepared lattice (obtained from the mainframe preparation network), no other classical data is ever transmitted to or from the user. This implies that even if an eavesdropper successfully taps into the quantum channel and entangles their own qubits to the cluster they will not know the basis the user chooses to measure in or have access to the classical error correction record. While an eavesdropper could employ a denial of service attack, the ability to extract useful information from the quantum channel is not possible without access to the classical information record measured by the client.

A second benefit to the secure model is that the client has ultimate control of whether their portion of the lattice generated by the host remains entangled with the larger global lattice of the mainframe. Performing $\sigma_{z}$ basis measurements on any photon within the cluster simply disentangles it from the lattice. Hence if the mainframe transmits a partial section of the generated lattice to the client, they simply perform $\sigma_{z}$ basis measurements on all photons around the edge of their partitioned allotment and they are guaranteed that neither the host and/or other users sharing the mainframe lattice can interact their portion of the lattice with the clients allotted section. This severing of the users sublattice from the mainframe would generally be recommended. If the sub-lattice is still linked to the mainframe, error correction procedures would need to be coordinated with the mainframe and classical data continually exchanged. This is due to the fact that error chains are able to bridge the region between user and host when links remain in-tact.

When a user has completed their task they have the option of making their results available to the global lattice, either to be utilized again or shared with other users. If the client does not wish to share the final quantum state of their algorithm, they measure all defect qubits and restore their portion of the lattice to a defect free state. If however, they wish to make available a non-trivial quantum state to the mainframe, then after their quantum algorithm is completed they can cease to measure the photons on the boundary of their allotted lattice. Once the client logs off the system, the quantum state of the defect qubits within this lattice will remain (provided the mainframe automatically continues measuring the sub-lattice to enact identity operations). Consequently, at a later time, the original user may de- 
cide to log onto the system again, or a second user may choose to $\log$ on that sub-lattice and continue to manipulate the stored data as they see fit (note that it is assumed that the global lattice is of sufficient size to allow for significant error protection and hence long term information storage). Additionally, this same methodology can be utilized to allow different users to interact quantum states. As with the previous case, two users may decide to perform independent, private, quantum algorithms up to some finite time and then interact data. Each user then ceases severing the connections to the global lattice and receives half an encoded Bell state from the mainframe, allowing for the implementation of teleportation protocols.

\section{Resource costs}

Although the preparation of a large 3D cluster lattice with photonic chips has been examined [5], how to partition resources for an optimal, multi-user device is a complicated networking problem. At this stage we will simply present an example partition structure for the resource lattice, hopefully demonstrating some of the essential features that would be needed for this model. We will approach this analysis with some basic numerical estimates to give an idea of the resource costs and physical lattice sizes for a mainframe device.

The HPQC mainframe will consist of two regions, an outer region corresponding to user partitions and an inner region which we will denote as scratch space. The scratch space will be utilized to for two primary tasks. The first is to provide logical Bell states to individual users in order to interact quantum information, the second is to distill and provide the high fidelity logical ancillae states $|A\rangle=(|0\rangle+i|1\rangle) / \sqrt{2}$ and $|Y\rangle=(|0\rangle+\exp (i \pi / 4)|1\rangle) \sqrt{2}$ which are needed to enact non-trivial single qubit rotations that cannot be directly implemented in the topological cluster model [11]. Purifying these states is resource intensive and as these states are required often for a general quantum algorithm it would be preferable to have an offline source of these states which does not consume space on the user partitions.

It should be stressed that the size of the scratch space lattice will be heavily dependent on the fundamental injection fidelity of these non-trivial ancilla states and consequently the amount of required state distillation [18]. This illustrative partitioning of the mainframe lattice, shown in Fig. 3, allocates a scratch space of $1000 \times 1000$ cells for each user region (effectively another computer the of the same size). In general, state distillation of ancilla states requires a large number of low fidelity qubits and distillation cycles and users will require a purified ancilla at each step of their computation [19]. Therefore, the scratch space could be sig-

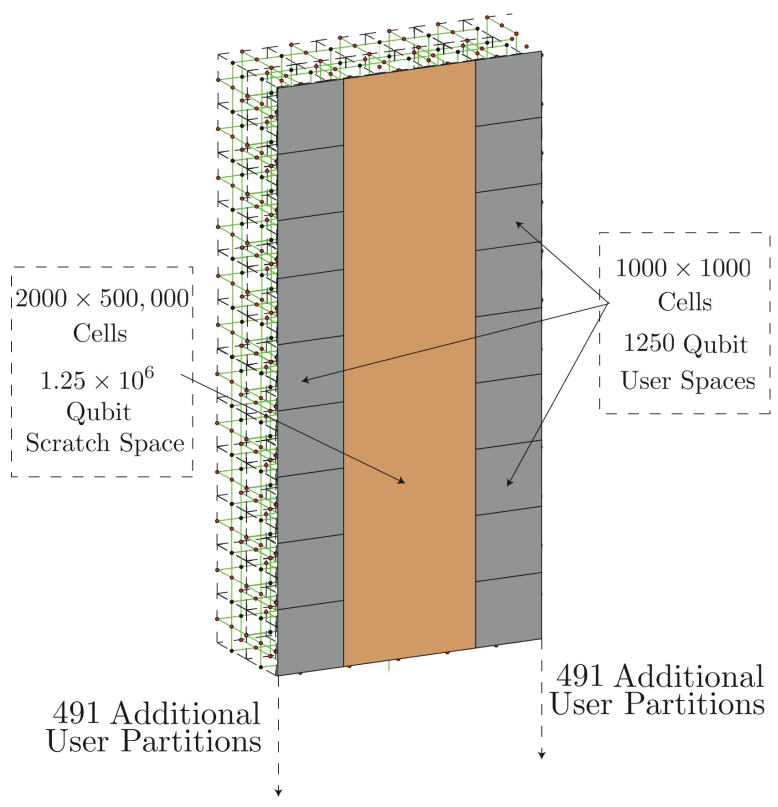

Fig. 3 Illustrated is an example partitioning of the global $3 \mathrm{D}$ lattice for a HPQC mainframe. This global lattice measures $4000 \times 500,000$ unit cells and requires approximately $7.5 \times 10^{9}$ photonic chips to prepare. If utilized as a single cluster computer, 2.5 million logical qubits are available with sufficient topological protection for approximately $10^{16}$ time steps (where a time step is defined as the measurement of a single layer of unit cells, corresponding approximately to $10^{11}$ logical, non-Clifford group operations [5]).

nificantly larger than each user partition. This does not change the general structure of the lattice partitioning, instead the width of the central scratch region is enlarged with user partitions still located on the boundaries. The primary benefit of requiring the mainframe to prepare purified ancilla is dynamical resource allocation, performed at the software level by the mainframe. By allowing the mainframe to prepare all distilled ancilla it is able to adjust the user/scratch partition structure to account for the total number of users and the required preparation rate of distilled states.

Based on this partitioning of the mainframe lattice we can illustrate the resource costs through a basic numerical estimate. As shown in [5], under reasonable physical assumptions, a large scale topological computer capable of running for approximately $10^{16}$ time steps (a time step is defined as the measurement of a single layer of unit cells, corresponding approximately to $10^{11}$ logical, non-Clifford group, operations [5]) requires approximately 3000 photonic chips per logical qubit, measuring $20 \times 40$ cells in the lattice. We therefore allocate each user a square region of the overall lattice measuring $1000 \times 1000$ unit cells, containing $50 \times 25$ 
logical qubits and requiring approximately $3.75 \times 10^{6}$ photonic chips to prepare. Additionally we consider a HPQC mainframe of sufficient size to accommodate 1000 individual user regions of this size with a scratch space two user regions wide and 500 user regions deep. Hence, this HPQC will need to generate a rectangular lattice measuring $4000 \times 500,000$ cells and require of order $7.5 \times 10^{9}$ photonic chips to prepare.

This may seem like a extraordinary number of devices to manufacture and incorporate into a large scale lattice generator, but one should recognise the enormous size of this mainframe. The partition structure is determined at the software level, no changes to the lattice preparation network is required to alter the structure of how the lattice is utilized. Hence, if desired, this mainframe can be utilized as a single, extremely large, quantum computer, containing 2.5 million logical qubits, with topological protection for approximately $10^{11}$ logical, non-Clifford operations [5], more than sufficient to perform any large scale quantum algorithm or simulation ever proposed.

\section{Conclusion}

In conclusion, we have introduced the concept of the High Performance Quantum Computer, where a massive 3-dimensional cluster lattice is utilized as a generic resource for multiple-user quantum information processing. The architectural model of 3D topological clusters in optics allows for the conceptual scaling of a large topological cluster mainframe well beyond what could theoretically be done with other architectures for QIP. As an example we illustrated a possible lattice partitioning of the mainframe system. This partition, while not optimal, shows some of the necessary structures that would be required for multi-user quantum computing. With this partition structure we were able to estimate the number of photonic chips required to construct a mainframe device. The construction of approximately 7.5 billion photonic chips leads to an extraordinary large multi-user quantum computer. While this is certainly a daunting task, this sized computer would represent the ultimate goal of QIP research that began in the late 1970's.

\section{Acknowledgements}

The authors thank A.G. Fowler, N. Lütkenhaus, A. Broadbent and T. Moroder for helpful discussions. The authors acknowledge the support of MEXT, JST, HP, the EU project HIP and the FIRST project.

\section{References}

[1] D. Kielpinski, C. Monroe, and D. J. Wineland, "Architecture for a large-scale ion-trap quantum computer," Nature, vol.417, pp.709-711, 2002.
[2] J. M. Taylor et. al., "Fault-tolerant architecture for quantum computation using electrically controlled semiconductor spins," Nature Phys., vol.1, pp.177-183, 2005.

[3] L. C. L. Hollenberg, A. D. Greentree, A. G. Fowler, and C. J. Wellard, "Two-dimensional architectures for donor-based quantum computing," Phys. Rev. B, vol.74, 045311, 2006.

[4] A. G. Fowler et. al., "Long-range coupling and scalable architecture for superconducting flux qubits," Phys. Rev. $B$, vol.76, 174507, 2007.

[5] S. J. Devitt et. al., "Architectural design for a topological cluster state quantum computer," New. J. Phys., vol.11, 083032, 2009.

[6] R. Stock and D. F. V. James, "Scalable, HighSpeed Measurement-Based Quantum Computer Using Trapped Ions," Phys. Rev. Lett., vol.102, 170501, 2009.

[7] R. van Meter, T. D. Ladd, A. G. Fowler, and Y. Yamamoto, "Distributed Quantum Computer Architecture Using Semiconductor Nanophotonics," Int. J. Quant. Info., vol.8, pp.295-323, 2010.

[8] D. A. Herrera-Marti, A. G. Fowler, D. Jennings, and T. Rudolph, "Photonic implementation for the topological cluster-state quantum computer," Phys. Rev. A., vol.82, 032332, 2010.

[9] N. Cody Jones et. al., "A Layered Architecture for Quantum Computing Using Quantum Dots," arXiv:1010.5022, 2010.

[10] R. Raussendorf and J. Harrington, "Fault-Tolerant Quantum Computation with High Threshold in Two Dimensions," Phys. Rev. Lett., vol.98, 190504, 2007.

[11] R. Raussendorf, J. Harrington, and K. Goyal, "Topological fault-tolerance in cluster state quantum computation," New J. Phys., vol.9, p.199, 2007.

[12] A. G. Fowler and K. Goyal, "Topological cluster state quantum computing", Quant. Inf. Comp., vol.9, no.9\&10, pp.721-738, 2009.

[13] S. J. Devitt et. al., "Photonic module: An on-demand resource for photonic entanglement," Phys. Rev. A., vol.76, 052312, 2007.

[14] R. Raussendorf and H. J. Briegel, "A One-Way Quantum Computer," Phys. Rev. Lett., vol.86, p.5188, 2001.

[15] P. Villoresi et. al., "Experimental verification of the feasibility of a quantum channel between space and Earth," New. J. Phys., vol.10, 033038, 2008.

[16] R. Ursin et. al. Proc. 2008 Microgravity Sciences and Process Symposium (2008); SECOQC, Project www.sqcoqc.net.

[17] W. J. Munro et. al., "From quantum multiplexing to high-performance quantum networking," Nature Photonics, vol.4, pp.792-796, 2010.

[18] S. Bravyi and A. Kitaev, "Universal quantum computation with ideal Clifford gates and noisy ancillas," Phys. Rev. A., vol.71, 022316, 2005. 
[19] A. G. Fowler, "Towards Large-Scale Quantum Computation," quant-ph/0506126, 2005.

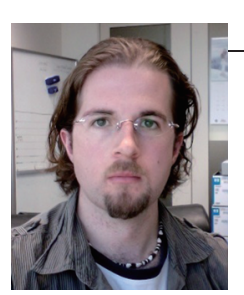

\section{Simon J. DEVITT}

Simon J. DEVITT is a Fellow at the National Institute for Informatics in Tokyo Japan. His research has been heavily focussed on the architectural construction of both quantum computing systems and communication networks and delving into what could be called "Quantum Engineering". He received his Ph.D from the centre for quantum computing technology in Australia in 2008.

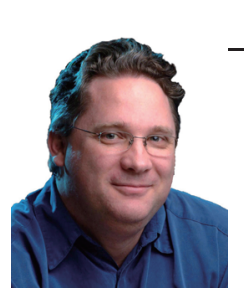

\section{William J. MUNRO}

William J. MUNRO is a research scientist within NTT Basic Research Laboratories in Japan. His current interests focus around practical implementations for optical and solid- state quantum hardware, generation of optical nonlinearities, characterization of quantum states and processes, novel quantum-communication protocols and quantum metrology.

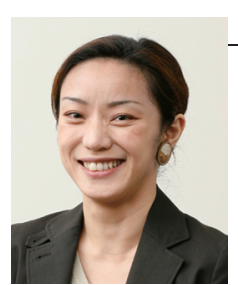

\section{Kae NEMOTO}

Kae NEMOTO is a Professor in the Principles of Informatics Research Division at the National Institute of Informatics, Tokyo, Japan. She heads the Quantum Information Science Theory Group and is a leader in the Japanese-France Laboratory for Informatics. Her research interests and efforts are currently focused around the implementation of quantum devices, architectures for large-scale QIP, quantum/atom optics and quantum nonlinear dynamics. 